\title{
Wirtschaftlichkeitsverfahren der santésuisse nach der ANOVA-Methode
}

\author{
Mirjam D’Angelo ${ }^{a}$, Paul Rhyn ${ }^{b}$ \\ a Projektleiterin Wirtschaftlichkeits- \\ verfahren \\ b Leiter Ressort Kommunikation
}

Der Entscheid des eidgenössischen Versicherungsgerichts vom 9. Oktober 2006 fordert die Gesamtbetrachtung (direkte und veranlasste Kosten) bei der Beurteilung der Wirtschaftlichkeit (siehe dazu [2]). santésuisse hat mit der ANOVAMethode die Gesamtkostenbetrachtung eingeführt (Statistikjahr 2004), damit werden implizit auch die Medikamentenkosten (direkt und veranlasst) der Onkologen kontrolliert.

Der Bericht von Jürg Nadig erweckt den falschen Eindruck, dass allein die ANOVA-Methode der santésuisse über Wirtschaftlichkeit und Unwirtschaftlichkeit von ambulanten Leistungen bei Ärzten entscheidet. Der Ablauf ist um einiges differenzierter. Um der Komplexität der Thematik gerecht zu werden, umfasst die Wirtschaftlichkeitsprüfung von santésuisse einen mehrstufigen Prozess (Abb. 1).

Mit der ANOVA-Methode von santésuisse erfolgt eine statistische Vorselektion. Dieser erste Schritt verlangt nach einer statistischen Methode, die für alle Ärzte in der gleichen Form anwendbar und unabhängig von der Fachrichtung

\section{Abbildung 1}

Prozessablauf der Wirtschaftlichkeitsverfahren der santésuisse.

$$
\begin{aligned}
& \text { dank Wirtschaftlichkeitsverfahren? } \\
& \text { Schweiz Ärztezeitung. } \\
& \text { 2008;89(20):855-60. } \\
& 2 \text { Kuhn Hp. Es gilt (endlich) die } \\
& \text { Gesamtkostenbetrachtung. } \\
& \text { Schweiz Ärztezeitung. } \\
& \text { 2006;87(50):2165-6. } \\
& 3 \text { D'Angelo M, Kraft P. Amstutz R. } \\
& \text { Neue statistische Methode für die } \\
& \text { Wirtschaftlichkeitsprüfung entbin- } \\
& \text { det Ärzte teilweise von Beweislast. } \\
& \text { Schweiz Ärztezeitung. } \\
& \text { 2005;86(31):1849-50. }
\end{aligned}
$$

Nadig J. Verdeckte Rationierung

Korrespondenz:

lic. oec. publ. Paul Rhyn

santésuisse

Römerstrasse 20

CH-4502 Solothurn

Tel. 0326254216

Fax 0326254151

paul.rhyn@santesuisse.ch ist. Es werden also Besonderheiten getestet und berücksichtigt, die flächendeckend für alle Ärzte in überprüfbarer Qualität vorliegen. Heute wird der Einfluss von Alter und Geschlecht der Patienten auf die Kosten untersucht und gegebenenfalls korrigiert. Zusätzlich wird auch den kantonalen Gegebenheiten Rechnung getragen (Korrekturfaktor Kanton), denn die ANOVA-Methode ermöglicht einen gesamtschweizerischen Vergleich. Je stärker ein Faktor (Alter, Geschlecht, Kanton) auf die Kosten wirkt, desto stärker fällt die Korrektur aus. Die beiden Faktoren Alter und Geschlecht der Patienten haben in den jeweiligen Facharztgruppen einen unterschiedlichen Effekt auf die Kosten, deshalb werden sie individuell pro Facharztgruppe korrigiert. Die Forderung von Jürg Nadig, das Geschlechterverhältnis als Besonderheit zu berücksichtigen, wird mit der ANOVAMethode somit bereits erfüllt (weitere Details zur ANOVA-Methode siehe [3]).

Weitere Besonderheiten werden in einem zweiten Schritt (Expertenbeurteilung) ermittelt und individuell berücksichtigt. Es wäre ineffizient, facharztspezifische Besonderheiten bereits im ersten Schritt mit der ANOVA-Methode zu berücksichtigen. Nebst den individuellen Besonderheiten der Onkologen müssten auch diejenige aller anderen Fachrichtungen im ANOVA-Modell Berücksichtigung finden. Ein solch komplexes Modell wäre aufgrund der resultierenden Wechselwirkungen der vielen Faktoren nicht mehr interpretierbar und somit völlig unadäquat für die Wirtschaftlichkeitskontrolle.

santésuisse ist sich bewusst, dass innerhalb der jeweiligen Facharztgruppen verschiedene Populationen existieren, die sich auf die Fallkosten auswirken, wie das bei den Onkologen der Fall ist. Im Rahmen der Expertenbeurteilung wird deshalb zusätzliches Statistikmaterial wie Originalrechnungen des betroffenen Arztes (vom Versicherer) oder verrechnete Tarifpositionen für die weitere Beurteilung herangezogen.

Da die Krankenversicherer aber, im Gegensatz zum Leistungserbringer selbst, nicht über die vollständige Information verfügen, ist in einigen Fällen auch ein Warnbrief oder ein Gespräch ange- 
zeigt, um einen umfassenden Überblick zu erhalten. Dieser weitere Schritt sichert die von Jürg Nadig geforderte individuelle Berücksichtigung von Besonderheiten wie Therapiestrategie, Praxisstruktur, Case-Mix usw. die mit einer statistischen Methode heute nicht abgedeckt werden können.

Fazit: Die ANOVA-Methode eignet sich als statistisches Instrument im Rahmen der Wirtschaftlichkeitskontrolle und in Kombination mit der anschliessenden individuellen Beurteilung. Sie erfüllt die Anforderung der Anwendbarkeit für alle Facharztgruppen und berücksichtigt diejenigen Besonderheiten, die flächendeckend und in überprüfbarer Qualität für alle Ärzte vorliegen.

Den von Jürg Nadig geschilderten Besonderheiten der Onkologen wird mit dem vorgestellten Prozessablauf der Wirtschaftlichkeitsverfahren der santésuisse genügend Rechnung getragen, um die Wirtschaftlichkeit beurteilen zu können. Diese Feststellung kann auf alle Facharztgruppen übertragen werden, da bei allen Facharztgruppen dieser mehrstufige Prozess angewendet wird.

\section{Replik auf die Stellungnahme von santésuisse}

\section{Jürg Nadig}

Korrespondenz:

Dr. med. Jürg Nadig Bannhaldenstrasse 7 CH-8180 Bülach

juerg.nadig@hin.ch santésuisse geht davon aus, die «statistische Vorselektion [...] [verlange] nach einer statistischen Methode, die für alle Ärzte in der gleichen Form anwendbar und unabhängig von der Fachrichtung ist, [... wobei] der Einfluss von Alter und Geschlecht der Patienten auf die Kosten untersucht und gegebenenfalls korrigiert [...] und den kantonalen Gegebenheiten Rechnung getragen» werde.

Unsere Analyse der Kostenstruktur der medizinischen Onkologen zeigt nun aber zum einen, dass die Vorselektion eben gerade nicht unabhängig von der Fachrichtung erfolgen kann, sondern bereits im Rahmen der Vorselektion eine differenzierte Betrachtung innerhalb der Fachrichtung notwendig ist (Anteil Therapien vs. Nachkontrollen, Körperoberfläche, Caseload und Therapiestrategie). Nur so können vergleichbare homogene Kollektive gebildet werden. Zum andern sind gerade die von santésuisse gemachten Korrekturen zum Einfluss von Alter oder kantonalen Gegebenheiten in der Onkologie irrelevant, da ältere Patienten seltener intensiv chemotherapiert werden und die Medikamentenkosten, die 3/4 der Behandlungskosten aus-machen, in allen Kantonen gleich sind. Da sich die Höhe der Rückforderung aber auf die im Rahmen der ANOVA-Methode bestimmte mittleren Kosten abstützt, wird entgegen der Behauptung von santésuisse die ANOVA-Methode mehr als ein statistisches Instrument der Vorselektion. Würden die verschiedenen Kollektive mit verschiedenen Kostenniveaus innerhalb einer Fachgesellschaft bereits in der Vorselektion berücksichtigt, wären keine unverhältnismässigen Rückforderungsklagen mehr möglich. Die
Onkologen könnten nicht mehr mit falsch berechneten Rückforderungen zu einer aussergerichtlichen Vereinbarung gezwungen werden, um nicht Konkurs zu gehen.

Gerade die von uns vorgeschlagene differenzierte Betrachtung bei der Vorselektion durch die Analyse von homogenen Kollektiven wäre wesentlich effizienter, da die Mehrzahl der Expertenbeurteilungen wegfielen. Bei korrekter Stratifizierung streuen die Kosten bei den Onkologen, die die Therapien selbst verabreichen und nicht an ein Spitalambulatorium delegieren, wie bei andern Kollektiven in der Regel innerhalb einer Bandbreite von 80 bis 130\% (in Absolutwerten z.B. zwischen 5000 und 7000 Franken), während das Mittel der Fallkosten für die ganze Fachgesellschaft z. B. bei 2500 (2000-3300) Franken liegen mag. Aus der Differenz der oberen Grenze der Fallkosten der Fachgesellschaft und den Fallkosten des Onkologen multipliziert mit der Anzahl Fälle errechnet santésuisse dann die Höhe der Rückforderung (in diesem Beispiel: [6000 - 3300] × 300 [Anzahl] Fälle = 810000 Franken).

Die Vorstellung von santésuisse, bereits das Kollektiv der Fachärzte garantiere eine homogene Verteilung der Kosten greift zu kurz. Leider ist die Wirtschaftlichkeitsprüfung komplexer als santésuisse es sich wünscht. Dieser Wunsch kann aber nicht zur Rechtfertigung einer undifferenzierten Anwendung der ANOVA-Methode dienen. In einem Rechtsstaat kann der Richter auch nicht Notrecht einführen und summarische Verfahren anordnen, nur weil er der Komplexität der Situation nicht mehr gewachsen ist. 
Unsere Feststellung, die ANOVA-Methode erfasse unwirtschaftliche Ärzte und Ärzte mit unwirksamen Behandlungsstrategien nicht und sei zudem willkürlich, weil sie nur Ärzte prüfe, die wegen des Einsatzes zugelassener wirksamerer, aber teurerer Therapien höhere Fallkosten verursachen (Beispiel Kolorektalkarzinom), kann santésuisse nicht entkräften.

santésuisse anerkennt zwar, dass «den Krankenversicherern zur Zeit aber (noch) nicht alle Informationen systematisch als Datensammlung zur Verfügung» stehen. Ungeachtet dessen hat sie aber in einzelnen Kantonen gegen Onkologen Wirtschaftlichkeitsklagen in existenzbedrohender Höhe eingereicht, mit der offen deklarierten Strategie, die Rückforderungen so hoch zu veranschlagen, dass der Arzt bei einem Gerichtsentscheid zu seinen Ungunsten wirtschaftlich ruiniert werde, da anschliessend auch für die folgenden Jahre Rückforderungen in gleicher Höhe rechtlich einverlangt würden. Das Ziel dieses Vorgehens ist es dann, über eine aussergerichtliche Einigung zumindest einen Teil der eingeklagten Summe zurückzuerhalten, ohne dass die Rechtmässigkeit der Forderung je von einem Richter beurteilt worden wäre. Da sich solche Rechtshändel über Jahre erstrecken können und die Rückforderungen wegen der hohen indirekten Kosten zwischen einer halben und einer Million Franken pro Jahr betragen, benötigte der eingeklagte Arzt ein finanzielles Polster von mehreren Millionen Franken, um einen solchen Prozess überhaupt durchstehen zu können, zumal in der Onkologie die hohen Kosten ja durch verabreichte Medikamente zustande kommen, die entweder rezeptiert werden oder mit einer sehr geringen Marge, die das Risiko der Rückforderung durch santésuisse in keiner Weise abdeckt, selbst verabreicht werden.

Unsere Fachgesellschaft machte santésuisse bereits 2006 auf diese Mängel bei der Anwendung der ANOVA-Methode zur Beurteilung der Onkologen aufmerksam. Wir vereinbarten mit santésuisse, gemeinsam die unterschiedlichen Profile der medizinischen Onkologen auszuarbeiten, um die Vorselektion wirksamer und gerechter durchzuführen. santésuisse verschob dieses Projekt kurzfristig aus «Ressourcenüberlegungen» auf Frühjahr/Sommer 2007 mit der Zu- sage, dass die verantwortlichen Experten auf die von uns aufgebrachte Problematik aufmerksam gemacht würden. santésuisse empfahl sogar den Experten, die Onkologen für diese Jahre zurückhaltend zu beurteilen. Offenbar beginnt auch santésuisse daran zu zweifeln, dass ihre Anwendung der ANOVA-Methode wirklich diejenigen Besonderheiten berücksichtigt, die notwendig sind, um die Wirtschaftlichkeit der Onkologen zu prüfen. Trotzdem hat santésuisse die Rückforderungsverfahren nicht eingestellt.

Keine Stellung nimmt santésuisse zu unseren Bedenken, dass die von ihr angewendete Form der Wirtschaftlichkeitsprüfung Tumorkranke dem Risiko aussetzt, dass ihnen aus Kostengründen wirksamere, aber teurere Therapiestrategien vorenthalten werden aus Angst vor Rückforderungsklagen durch santésuisse, was einer verdeckten Rationierung gleichkäme.

Die Stellungnahme von santésuisse zeigt, dass sich die komplexe Wirklichkeit im Gesundheitswesen nicht mit der ANOVA-Methode und Wirtschaftlichkeitsverfahren steuern lässt. Die ANOVA-Methode eignet sich auch in Kombination mit der anschliessenden individuellen Beurteilung nicht als statistisches Instrument im Rahmen der Wirtschaftlichkeitskontrolle. Die Behauptung von santésuisse, «die ANOVA-Methode [erfülle] die Anforderung der Anwendbarkeit für alle Facharztgruppen und [berücksichtige] diejenigen Besonderheiten, die flächendeckend und in überprüfbarer Qualität für alle Ärzte vorliegen», trifft offensichtlich nicht zu. Die Willkürlichkeit der individuellen Beurteilung zeigt sich bereits darin, dass in einem Kanton bei vergleichbaren Fallkosten eine Rückforderungsklage eingereicht wird, während in einem andern Kanton dem Onkologen beschieden wird, seine Kosten seien nachvollziehbar und nicht zu beanstanden.

Zur Beurteilung der Wirtschaftlichkeit braucht es im Interesse des Patienten eine Monitorisierung der Behandlungsqualität und die Erfassung der Vollkosten innerhalb einer integrierten Behandlungskette. Nur so wird verhindert, dass den Schwerkranken wirksamere, aber teurere Therapien aus Angst vor Wirtschaftlichkeitsverfahren implizit vorenthalten werden. 